\title{
Zoledronic acid for osteoporosis treatment-a matter of time
}

Adherence to treatment is a considerable concern in all areas of medicine. With respect to patients with osteoporosis, an annual intravenous infusion of zolendroic acid (ZOL) increases bone mineral density (BMD) and reduces bone turnover-in an effort to reduce fracture risk-but also results in high adherence rates owing to the lack of dependency on patients to selfadminister doses at frequent intervals. Two studies, published in the Journal of Bone and Mineral Research, now shed further light on the efficacy and duration of action of $\mathrm{ZOL}$ in patients with osteoporosis.

\section{$44 . . .74 .2 \%$ of participants ... [preferred an] annual intravenous infusion of $\mathrm{ZOL}$... in comparison to ... weekly oral alendronate 77}

Eric Orwoll and colleagues have demonstrated that an annual intravenous infusion of ZOL, known to be a successful therapy in postmenopausal women, is also effective in increasing BMD and reducing bone turnover markers in men with osteoporosis, and suggest it might help improve adherence rates by replacing weekly oral doses of alendronate. "In comparison to women, osteoporosis in men is very much under-studied and there are fewer trials of potential therapies," explains Orwoll. "There is also very little information about the comparative effectiveness of various agents."
In a multicenter, double-blind, doubledummy trial, the researchers randomized men with osteoporosis to receive either an annual intravenous infusion of $5 \mathrm{mg}$ ZOL ( $n=154)$ or a weekly oral $70 \mathrm{mg}$ alendronate tablet $(n=148)$ for 24 months. Both treatments increased lumbar spine, total hip, femoral neck and trochanter $\mathrm{BMD}$, and the researchers established the noninferiority of ZOL in comparison with alendronate. Both ZOL and alendronate reduced bone turnover markers to a similar extent at 12 and 24 months of treatment. Following the completion of the trial, $74.2 \%$ of participants indicated a preference for annual intravenous infusion of ZOL compared with 15.3\% who preferred weekly oral alendronate. Despite the participants' clear preference for ZOL, Orwoll comments that "the salient issue is to what extent fractures are reduced with ZOL therapy in men, but that would take very large trials that are unlikely to be performed."

In a separate study, Andrew Grey and co-workers determined that the beneficial effects of a single intravenous dose of $5 \mathrm{mg} \mathrm{ZOL}$ are sustained for at least 3 years in postmenopausal women with osteopenia (a precursor to osteoporosis), and so repeating this dosage at 1-year intervals might be surplus to requirement. The investigators randomized 50 postmenopausal women with osteopenia to receive either a single intravenous dose of $5 \mathrm{mg} \mathrm{ZOL}$ or placebo. At 3-year follow-up, women in the ZOL group sustained an approximately $40 \%$ reduction in bone turnover markers, and a $6.8 \%, 4.0 \%$ and $2.0 \%$ increase in BMD with respect to lumbar spine, total hip and total body, respectively, in comparison with those receiving placebo. However, the authors stress that clinical trials are needed to assess whether a dosing interval of ZOL of more than 1 year reduces fracture risk.

Taken together, these studies suggest that an annual intravenous infusion of ZOL is equally as effective as other treatment options for patients with osteoporosis or osteopenia. Moreover, the chance of patient noncompliance is likely to be reduced because, depending on the patient, an infusion is required only once every 1-3 years. Indeed, Grey and colleagues suspect that the effects of ZOL might extend beyond 3 years. "Since there was still clear evidence of antiresorptive activity at the end of the study, we have extended the protocol for a further 2 years, in order to clearly determine the duration of antiresorptive activity of a single dose of the drug," comments Grey.

\section{Rowan Higgs}

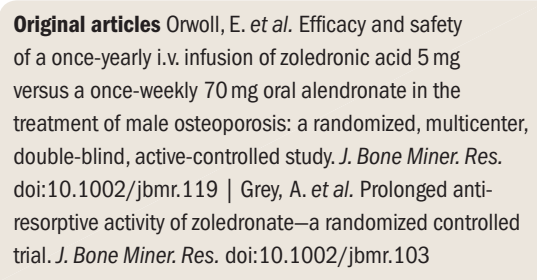

